\title{
ON THE HEATING OF A TWO-DIMENSIONAL SLAB IN A MICROWAVE CAVITY: APERTURE EFFECTS
}

\author{
T. R. MARCHANT' and B. LIU ${ }^{\prime}$ \\ (Received 10 November, 1998; revised 22 February, 1999)
}

\begin{abstract}
The steady-state heating of a two-dimensional slab by the $T E_{30}$ mode in a microwave cavity is considered. The cavity contains an iris with a variable aperture and is closed by a short. Resonance can occur in the cavity, which is dependent on the short position, the aperture width and the temperature of the heated slab.

The governing equations for the slab are steady-state versions of the forced heat equation and Maxwell's equations while fixed-temperature boundary conditions are used. An Arrhenius temperature dependency is assumed for both the electrical conductivity and the thermal absorptivity. Semi-analytical solutions, valid for small thermal absorptivity, are found for the steady-state temperature and the electric-field amplitude in the slab using the Galerkin method.

With no-iris (a semi-infinite waveguide) the usual S-shaped power versus temperature curve occurs. As the aperture width is varied however, the critical power level at which thermal runaway occurs and the temperature response on the upper branch of the S-shaped curve are both changed. This is due to the interaction between the radiation, the cavity and the heated slab. An example is presented to illustrate these aperture effects. Also, it is shown that an optimal aperture setting and short position exists which minimises the input power needed to obtain a given temperature.
\end{abstract}

\section{Introduction}

There is growing interest in the use of microwave radiation in a range of industrial processes such as drying, smelting, sintering, joining and welding. The main advantage of microwave heating over conventional convective heating methods is that the processing time can be reduced dramatically. Controlled uniform heating is difficult to achieve however, with thermal runaway occurring in some materials for a small increase in incident power.

\footnotetext{
'School of Mathematics and Applied Statistics, University of Wollongong, Wollongong, NSW, Australia.

(C) Australian Mathematical Society 2001, Serial-fee code 0334-2700/01
} 
The phenomenon of thermal runaway, due to the temperature dependence of the material properties, is analogous to that of the combustion of an exothermic chemical reactant or a flashover in a fire. Moreover, the power versus temperature relationship is described by the classical S-shaped curve of combustion theory, hence thermal runaway occurs when the temperature jumps from the low (cool) branch to the upper (hot) solution branch. With chemical combustion the upper (hot) branch owes its existence to the exothermic reaction term being limited at high temperatures; via either an Arrhenius-type reaction rate or the depletion of the reactant. The electricfield generated by the microwave radiation in the material has a similar role to that of the chemical reactant. In the case of microwave heating the upper branch is formed due to the temperature-dependent electrical conductivity quenching the electric-field amplitude in the material at high temperatures. Hence microwave heating has many close analogies with the combustion of chemical reactants.

The rapid increase in temperature associated with thermal runaway can have dramatic effects. For example, ceramics are normally sintered by heating for a long period of time in a conventional kiln. Processing inside a microwave cavity or waveguide can dramatically reduce the sintering time. However, thermal runaway can result in the sample being heated to its melting point, and hence destroyed. Other heating scenarios are also possible. For example, the processing temperature may lie on the upper branch in the region of multiple solutions; in this case feedback control may be needed to reach the desired steady-state temperature.

The equations governing the microwave heating of a material are Maxwell's equations, governing the propagation of microwave radiation through the material, and the forced heat equation, governing the heat absorption and the resultant heat diffusion. It is also necessary to calculate the electromagnetic field within the waveguide or microwave cavity, inside which the material is processed. The prediction of thermal runaway thus requires the numerical solution of Maxwell's equations within the material and the waveguide coupled with the solution of the forced heat equation inside the slab. For a realistic three-dimensional material this approach is computationally expensive and very slow in providing predictions of thermal runaway. Hence the development of approximate analytical models of microwave heating has generated a lot of interest in the last few years; the survey article of [1] details much of this work.

Liu and Marchant [6] considered the heating of a two-dimensional slab in a long rectangular waveguide propagating the $T E_{10}$ waveguide mode (see [3] for a definition of the $T E_{10}$ mode). The temperature dependency of the electrical conductivity and the thermal absorptivity was the Arrhenius law, while the other material properties were assumed constant. Approximate analytical solutions, valid for small thermal absorptivity, were found for the temperature and the electric-field amplitude using the Galerkin method. Examples were presented in the limits of small and large heat-loss and also for an intermediate case involving radiative heat-loss; an excellent 
comparison with numerical solutions was obtained in all cases. Also a control process was introduced and the transient heating of the slab was examined. It was shown that the choice of feedback parameters is important; inappropriate choices led to temperature overshoot or relaxation oscillations.

Kriegsmann [3] considered the small Biot-number heating of a ceramic slab in a $T E_{103}$ waveguide applicator including an iris with a variable aperture. The $T E_{103}$ waveguide applicator propagates the $T E_{10}$ mode and is three half-wavelengths long. In the small Biot-number limit the heat-loss through the slab boundaries is small, hence the temperature profile is nearly uniform. This allows the integration of the forced heat equation over the slab; the $\mathrm{S}$-shaped power versus temperature response curve is obtained. It was shown that the microwave cavity can be tuned by varying the aperture width, as the limit points at which bifurcation occurs and the temperature response of the upper branch both change. Hence there is an optimal aperture width which sinters the ceramic with minimum power. Moreover, varying the aperture width (and hence the S-shaped curve) allows a sintering temperature on the upper branch to be reached without feedback control.

In this paper the microwave cavity model of Kriegsmann [3], valid in the small Biot-number limit, and the long-waveguide model of Liu and Marchant [6], valid for arbitrary heat-loss, are melded and a microwave cavity model, valid in the large Biot-number limit, is presented. In Section 2 the steady-state governing equations are derived. The electromagnetic theory for the cavity, and the various simplifying assumptions, from Kriegsmann [3], are used. In Section 3 approximate analytical solutions for the electric-field amplitude and the temperature in the slab are developed, using the theory of Liu and Marchant [6]. In Section 4 some results are presented which show the effect of varying the aperture width. It is shown that an optimal aperture and short position exists which minimises the incident power required for a given processing temperature.

\section{The governing equations}

In this section the steady-state governing equations and boundary conditions for the temperature and electric-field amplitude in the slab are formulated. To achieve this the electric-field in the microwave cavity must be found. Figure 1 shows a sketch of the microwave cavity. The square slab completely fills the cross-section of the waveguide (between $y= \pm 1$ ) and lies between $x=q$ and $x=q+2$. The microwaves enter the cavity through the iris at $x=0$. The iris aperture has a width of $2(1-a)$, hence $a=0$ corresponds to no iris while for $a=1$ the aperture is shut. A short placed a distance $d$ beyond the slab, at $x=q+d+2$, closes the end of the waveguide. The short reflects the microwaves, which can cause resonance in the cavity. 


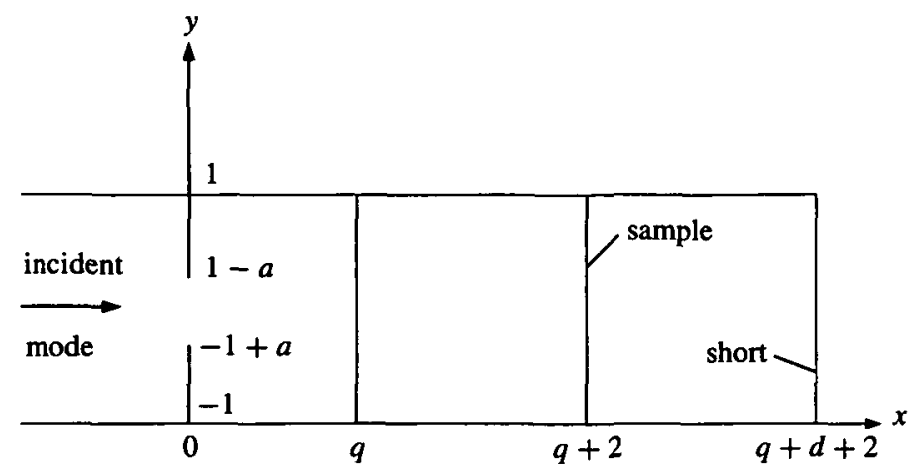

FIGURE 1. The slab and the microwave cavity.

For a two-dimensional waveguide, transverse electric $(T E)$ modes are appropriate, hence the electric field in the slab is given by

$$
\mathbf{E}=U(x, y) e^{-i \omega t} \mathbf{k},
$$

where the electric-field has been written as a steady-state amplitude modulated by the frequency. The governing equation for the electric-field amplitude in the slab is

$$
U_{x x}+U_{y y}+k_{1}^{2}(1+\sigma) U=0 .
$$

The parameters $k_{1}=\omega / c$ and $\sigma$ are the wavenumber and electrical conductivity respectively. The electric-field amplitude in the cavity is given by

$$
\begin{array}{ll}
\cos (\pi y / 2)\left(e^{i k_{r} x}+\rho e^{-i k_{r} x}\right), & x<0, \\
\cos (\pi y / 2) \psi\left(e^{i k_{r} x}+\gamma e^{-i k_{r} x}\right), & 0<x<q, \\
\cos (\pi y / 2) \tau\left(e^{i k_{r} x}-e^{-i k_{r}(x-2 q-2 d-4)}\right), & q+2<x<q+2+d,
\end{array}
$$

where

$$
2 k_{r}=\left(4 k^{2}-\pi^{2}\right)^{1 / 2}, \quad \pi<2 k<2 \pi,
$$

where $k$ is the wavenumber in free-space. An electric-field of amplitude unity is incident upon the iris. The coefficients $\psi$ and $\tau$ represent the electric-field amplitudes which are incident upon and transmitted through the slab respectively. The coefficients $\rho$ and $\gamma$ are reflection coefficients from the iris and slab respectively. Reflection from the short, at $x=q+d+2$, means that resonance can occur in the cavity with the electric-field amplitude incident upon the slab greater than unity $(|\psi|>1)$. The electric-field amplitudes (3) satisfy the wave equation ((2) with $\sigma=0$ and $k_{1}=k$ ) in the cavity and the boundary condition of zero electric-field amplitude at the waveguide walls and at the short. The wavenumber, $k_{r}$, is that of the lowest waveguide mode, $T E_{10}$. 
The wavenumber in free space, $k$, is chosen so that the wavenumbers of the higher waveguide modes are imaginary. This means that the higher modes are evanescent (they do not propagate) and hence can be ignored as long as the slab is sufficiently far away from the iris and the short.

Assuming continuity of the electric-field amplitude and its derivatives across the aperture gap in the iris gives

$$
\psi=\frac{1+r}{1-\gamma r},
$$

where $r$ is the reflection coefficient for a wave of unit amplitude incident upon an iris in a long empty waveguide. The approximation of Lewin [5] was used by Kriegsmann [3]. This, rescaled for a waveguide of width two, is

$$
r=\frac{-i \pi s}{2 k_{r}+i \pi s}, \quad \delta=\left(1-\frac{4 k^{2}}{9 \pi^{2}}\right)^{1 / 2}, \quad s=\tan ^{2}\left(\frac{\pi a}{2}\right)-\frac{3 \delta \sin ^{4}(\pi a / 2)}{1-\delta \cos ^{2}(\pi a / 2)} .
$$

The electric-field amplitude in the slab is given by (2) and the boundary conditions by

$$
\begin{array}{ll}
U_{x}+i k_{r} U=2 i k_{r} \cos (\pi y / 2), & \text { at } x=-1, \\
U_{x}+k_{r} \cot \left(k_{r} d\right) U=0, & \text { at } x=1, \\
U=0, & \text { at } y= \pm 1,
\end{array}
$$

which represent the reflection and transmission of the radiation at the leading and trailing edges of the slab plus zero electric-field amplitude at the waveguide walls. The electric-field amplitude has been scaled by the factor $\psi e^{i k_{r} q}$ and the $\mathrm{x}$-coordinate is scaled so that the slab now lies between $x= \pm 1$. The steady-state forced heat equation and boundary conditions for the slab are

$$
T_{x x}+T_{y y}+\beta f(T)|U|^{2}|\psi|^{2}=0, \quad T=0 \quad \text { at } x, y= \pm 1 .
$$

The heat source term comprises the thermal absorptivity $\beta f(T)$ and the square of the electric-field amplitude. The electrical conductivity $\sigma=\alpha f(T)$ and the thermal absorptivity have the same temperature dependency, as physically it is expected that energy lost by the microwave radiation is absorbed as heat. For simplicity the electricfield amplitude is represented by two terms. The first is $|U|^{2}$, which represents the electric-field amplitude in the slab, given a wave of amplitude unity is incident upon it. The second term, $|\psi|^{2}$, is due to the iris and does not appear in the governing equations describing the heating in a long rectangular waveguide (see Liu and Marchant [6]). The effect of the iris is to change the amplitude of the electric-field incident upon the slab. This term is implicitly temperature dependent, via the reflection coefficient $\gamma$. 
The other modification, which takes into account the short, is to the boundary condition for the electric-field amplitude at the trailing edge of the slab, $x=1$. Also a fixedtemperature boundary condition applies on the slab's edges. This represents the large Biot-number limit, in which the heat-loss from the slab is significant, and complements the small Biot-number theory of Kriegsmann [3].

\section{Approximate analytical solutions}

Liu and Marchant [6] developed approximate analytical solutions for the temperature and the electric-field amplitude of a two-dimensional slab in a long rectangular waveguide using the Galerkin method. Here this method is used to obtain approximate solutions for a two-dimensional slab in a microwave cavity, described by the equations (2), (6) and (7).

The Arrhenius law

$$
f(T)=1+20 e^{-1 / T},
$$

is used as the temperature dependency for both the thermal absorptivity and electrical conductivity of the slab. For many materials molecules occupy potential wells at low temperatures and cannot absorb microwave radiation. At higher temperatures however these molecules are freed and more easily absorb the radiation [2]. Hence a absorption law of the form (8) is appropriate as the probability of a molecule being in a given quantum state is given by the Gibbs distribution of statistical mechanics [4].

At the ambient temperature, by the Arrhenius law $f(0)=1$, as the temperature becomes large, $f(T) \rightarrow 21$. Hence it is bounded at large temperatures. The Arrhenius law is not amenable to analytical work, so a rational-cubic function, of the form

$$
f(T)=\frac{R_{1}(T)}{R_{2}(T)}, \quad \text { where } \quad R_{i}(T)=\sum_{j=0}^{3} r_{i, j} T^{j}, \quad i=1,2,
$$

is used to approximate it. Liu and Marchant [6] found the parameters to be

$$
\begin{aligned}
& r_{10}=r_{20}=1, \quad r_{11}=5.469, \quad r_{12}=-43.34, \quad r_{13}=224.91 \text {, } \\
& r_{21}=2.534, \quad r_{22}=8.255, \quad r_{23}=10.71 \text {, }
\end{aligned}
$$

by equating ( 8 ) and (9) in the limits of small and large temperatures and by using the method of least squares. The form of rational function (9) is chosen as it is bounded at high temperatures and contains sufficient free parameters to enable a good fit with the Arrhenius law to be obtained at all temperatures. Alternatively, a polynomial function could be used to approximate the Arrhenius law over a finite temperature range. The polynomial function would not be bounded at very high temperatures; this however would be outside the temperature range of physical interest. 
Generally, the Galerkin method requires that the exact solution be approximated by a sum of orthogonal basis functions. The parameters associated with the basis functions are found by evaluating averaged versions of the governing equations, weighted by the basis functions themselves. Here both the electric-field amplitude and the temperature are represented by one basis function only,

$$
\begin{aligned}
& T=C \phi_{1}, \quad \phi_{1}=\cos (\pi x / 2) \cos (\pi y / 2), \\
& U=\phi_{2}=\cos (\pi y / 2)(A \cosh (b x)+B \sinh (b x)),
\end{aligned}
$$

where $C$ and $b$ are parameters to be determined. The basis function for the electricfield amplitude is the exact solution for constant electrical conductivity. The basis function for the temperature is chosen from the series solution for the unforced heat equation. Note that the temperature profile is symmetric with a maximum $C$ at the slab's centre. This approximate solution will be valid when the thermal absorption is fairly small, which generally means the lower (cooler) branch of the S-shaped curve. Note however, that Liu and Marchant [6] found an excellent agreement between the semi-analytical and numerical solutions on both the lower and upper solution branches.

The first equality of (11) satisfies the fixed-temperature condition on the slab's edges while the boundary conditions (7) for the electric-field amplitude imply

$$
\begin{gathered}
A=A_{1} e^{b}+B_{1} e^{-b}, \quad B=A_{1} e^{b}-B_{1} e^{-b}, \quad \text { where } \\
A_{1}=2 i k_{r} Z e^{-2 b} D^{-1}, \quad B_{1}=-2 i k_{r} \hat{Z} e^{2 b} D^{-1}, \quad Z=k_{r} \cot \left(k_{r} d\right)-b, \\
\hat{Z}=k_{r} \cot \left(k_{r} d\right)+b, \quad D=\left(b+i k_{r}\right) Z e^{-2 b}-\hat{Z}\left(i k_{r}-b\right) e^{2 b} .
\end{gathered}
$$

Substituting the basis functions (11) into the governing equation for the electricfield amplitude (2), multiplying by the weight $\phi_{2}^{-1}$, and averaging over the slab gives the decay rate as

$$
\begin{aligned}
b & =\left[\left(\pi^{2} / 4\right)-k_{1}^{2}\left(1+i \alpha J_{1} / J_{2}\right)\right]^{1 / 2}, \\
J_{i} & =4 r_{i 0}+\frac{16 r_{i 1} C}{\pi^{2}}+r_{i 2} C^{2}+\frac{64 r_{i 3} C^{3}}{9 \pi^{2}}, \quad i=1,2,
\end{aligned}
$$

while applying the weight $\phi_{1}$ to the forced heat equation and averaging gives

$$
\beta=\frac{\pi^{2} C\left\langle g_{2}\right\rangle}{2|\psi|^{2}\left\langle g_{1}\right\rangle}, \quad g_{1}=\phi_{1} R_{1}\left(C \phi_{1}\right)\left|\phi_{2}\right|^{2}, \quad g_{2}=\phi_{1}{ }^{2} R_{2}\left(C \phi_{1}\right),
$$

where \langle\rangle refers to the average of the quantity over the slab. Expression (14) describes the S-shaped power $(\beta)$ versus temperature $(C)$ curve. In order to facilitate the calculation of the integral of $g_{1}$ in (14), the square of the electric-field amplitude is written

$$
\begin{aligned}
& \left|\phi_{2}\right|^{2}=\cos ^{2}(\pi y / 2)\left(a_{1} \cosh (2 u x)+b_{1} \cos (2 v x)\right), \quad \text { where } \\
& b=u+i v, \quad 2 a_{1}=A \bar{A}+B \bar{B}, \quad 2 b_{1}=A \bar{A}-B \bar{B} .
\end{aligned}
$$


The new parameters $a_{1}$ and $b_{1}$ are both real. The terms $u$ and $v$ are the real and imaginary parts of the decay rate $b$. Also note that the expression (15) contains only the symmetric terms of $\left|\phi_{2}\right|^{2}$ as the nonsymmetric terms integrate to zero in (14). The integrals of $g_{1}$ and $g_{2}$ are

$$
\begin{aligned}
\left\langle g_{1}\right\rangle= & r_{10} \frac{8}{3 \pi}\left(\frac{4 \pi a_{1} \cosh (2 u)}{16 u^{2}+\pi^{2}}+\frac{4 \pi b_{1} \cos (2 v)}{\pi^{2}-16 v^{2}}\right) \\
+ & r_{11} C \frac{1}{2}\left[a_{1}\left(\frac{\sinh (2 u)}{2 u}-\frac{2 u \sinh (2 u)}{4 u^{2}+\pi^{2}}\right)+b_{1}\left(\frac{\sin (2 v)}{2 v}+\frac{v \sin (2 v)}{2 \pi^{2}-2 v^{2}}\right)\right] \\
+ & r_{12} C^{2} \frac{32}{15 \pi}\left[a_{1}\left(\frac{3 \pi \cosh (2 u)}{16 u^{2}+\pi^{2}}-\frac{3 \pi \cosh (2 u)}{16 u^{2}+9 \pi^{2}}\right)\right. \\
& \left.+b_{1}\left(\frac{3 \pi \cos (2 v)}{\pi^{2}-16 v^{2}}-\frac{3 \pi \cos (2 v)}{9 \pi^{2}+16 v^{2}}\right)\right] \\
+ & r_{13} C^{3} \frac{3}{8}\left[a_{1} \sinh (2 u)\left(\frac{3}{8 u}-\frac{2 u}{4 u^{2}+\pi^{2}}+\frac{u}{8 u^{2}+4 \pi^{2}}\right)\right. \\
& \left.\quad+b_{1} \sin (2 v)\left(\frac{3}{8 v}+\frac{2 v}{\pi^{2}-4 v^{2}}-\frac{v}{8 \pi^{2}-8 v^{2}}\right)\right] \\
\left\langle g_{2}\right\rangle= & r_{20}+\frac{64 r_{21} C}{9 \pi^{2}}+\frac{9 r_{22} C^{2}}{16}+\frac{32^{2} r_{23} C^{3}}{15 \pi^{2}}
\end{aligned}
$$

The power versus temperature curve (14) can be calculated explicitly. For a given temperature maximum $C$ the decay rate $b$ can first be found from (13). Then the coefficients $A$ and $B$ in (12) and the parameters $a_{1}, b_{1}, u$ and $v$ from (15) can all be found. Next, the integrals of $g_{1}$ and $g_{2}$ are calculated from (16). Lastly, the reflection coefficient $\gamma=e^{2 i k_{r} q}\left(A_{1}+B_{1}-1\right)$ and the incident electric-field amplitude $|\psi|$ are determined.

\section{Results and discussion}

The common parameters used for the results are $k=k_{1}=2, k_{r}=1.238, \alpha=$ $5 \times 10^{-3}$ and $q=1$. The wavenumber $k_{r}$ of the $T E_{10}$ mode is chosen so that higher modes are evanescent, while the position of the slab is chosen so that it is far enough away from the iris to neglect these modes. The decay rate of the electric-field, $\alpha$, is chosen to be small. Figure 2 shows the steady-state temperature $C$ versus $\beta$ for various choices of aperture opening $a$ while Figure 3 shows the critical power level $\beta_{c}$ versus aperture opening $a$. The short position is at $d=2.62$. When there is no iris ( $a=0$ ) the cavity becomes a semi-infinite waveguide; see Liu and Marchant [6]. The semi-infinite waveguide is closed at one end (the short is at $x=q+d+2$ ) with the incident mode originating at $x=-\infty$. In this case the electric-field amplitude 


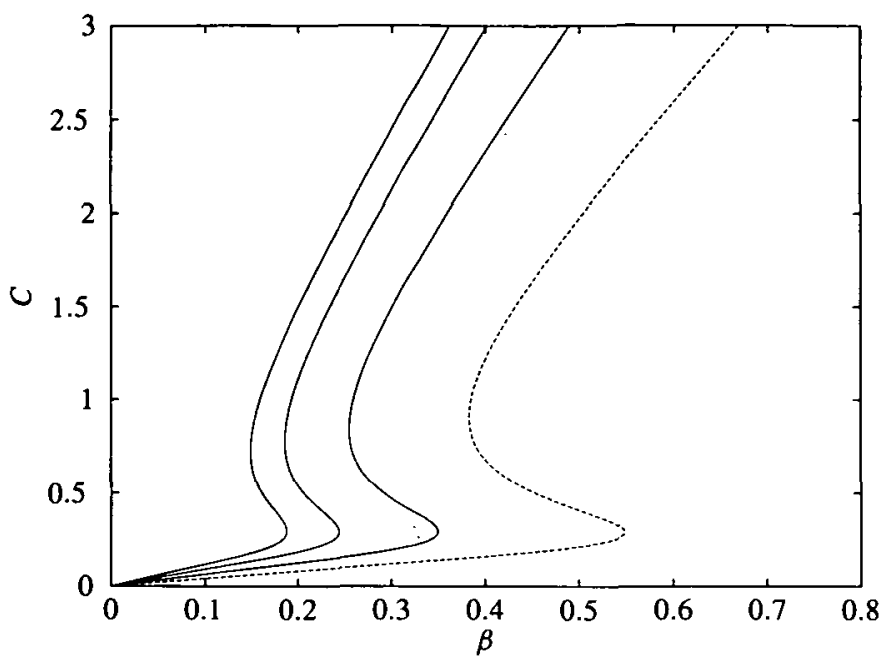

FIGURE 2. Temperature versus power curves for different aperture openings. The solid curves shown (from right to left) are for $a=0,0.3$ and 0.4 respectively while the dashed curve represents $a=0.6$.

incident upon the slab, $|\psi|$, is equal to unity. In the no-iris case the $S$-shaped response curve has a critical power level of $\beta=0.35$, at which the solution jumps from the cool to the hot branch. As the aperture is progressively narrowed the lower branch folds back and the critical $\beta$ is dramatically reduced (see Figure 3). Moreover, the power level $\beta$ needed to achieve a given temperature $C$ is reduced, hence the cavity is more efficient than in the no-iris case. This is due to resonance occurring within the cavity which increases the incident electric-field amplitude, $|\psi|$, beyond unity. Beyond a critical value of the aperture opening ( $a=0.44$ ) however, the cavity is detuned. The $S$-shaped response curve moves back to the right (see the dashed curve in Figure 2), the critical value $\beta_{c}$ increases (see Figure 3 ) and the cavity becomes less efficient than in the no-iris case.

The cavity effects described above are largely due to interaction between the cavity and the radiation. For a loss-less slab (with $\alpha=0$ ) the incident electric-field amplitude reaches a maximum of $|\psi|=1.42$ for $a=0.44$. The heated slab also interacts with the cavity, in this case detuning it somewhat. For example, when $C=3$, the maximum incident electric-field amplitude $|\psi|=1.16$, again at $a=0.44$. This detuning occurs because the decay rate $b$ changes the reflection coefficient $\gamma$ which in turn affects the incident electric-field amplitude $|\psi|$.

Varying the aperture width can also make the desired steady-state processing temperature more accessible. Consider a temperature ( $C=1.5$ say) which lies on the upper branch of the no-iris curve where multiple solutions exist. This point is not easily reached from the ambient temperature as the solution will tend to the lower steady-state solution. Normally feedback control is needed to achieve the upper branch 


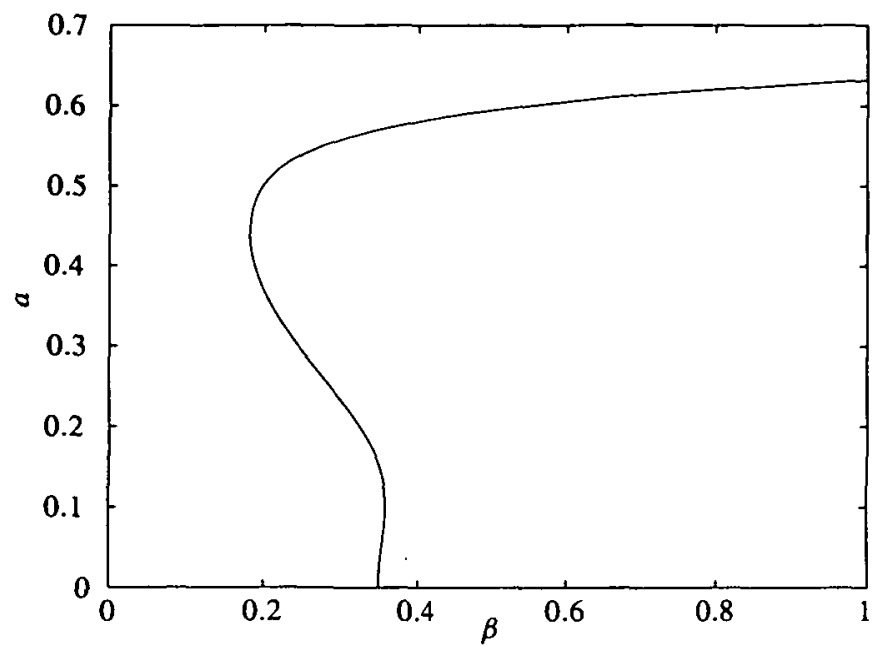

FIGURE 3. Critical power level versus aperture opening.

steady-state solution in this case; however if the aperture is chosen as $a=0.4$ the lower branch folds back and the desired solution can be reached without feedback control. The hotter steady-state solution could be reached without feedback control or aperture adjustment if the initial temperature of the slab is greater than some critical value. However, it would not be practical to initially heat the slab by conventional convective means before beginning microwave processing.

Figure 4 shows the power $\beta$ versus aperture opening $a$ for a fixed processing temperature $C=5$. Note that the solution is periodic with respect to short position; the solution is unchanged if the short is moved a multiple of $x=\pi k_{r}^{-1}=2.54$. This is due to the periodic term $\cot \left(k_{r} d\right)$ in (6). The first short position, $d=2.07$, corresponds to resonance in an empty cavity for a small aperture opening. In this case the incident electric-field amplitude $|\psi| \approx 100$ for $a=0.92$. This short position is chosen in a similar way to that in Kriegsmann [3]. Once the lossy slab is placed within the cavity however, detuning occurs with $|\psi|$ being much less than unity for large $a$. For smaller values of $a(<0.6)$ the power versus aperture opening curve is very nearly flat and there is no increased efficiency over the no-iris case.

The other short position, $d=2.62$, is chosen to minimise the incident power required for a cavity containing a slab at the desired temperature, $C=5$, over all possible choices of $a$ and $d$. This optimisation of the microwave heating process depends on two important effects; resonance within the cavity and slab placement (relative to the short). Kriegsmann [3] reports that these effects are subject to ongoing experimental work.

It can be seen that this new choice of short position makes the cavity less efficient when $a$ is large $(a>0.6)$. The first short position of $d=2.07$ was chosen to make 


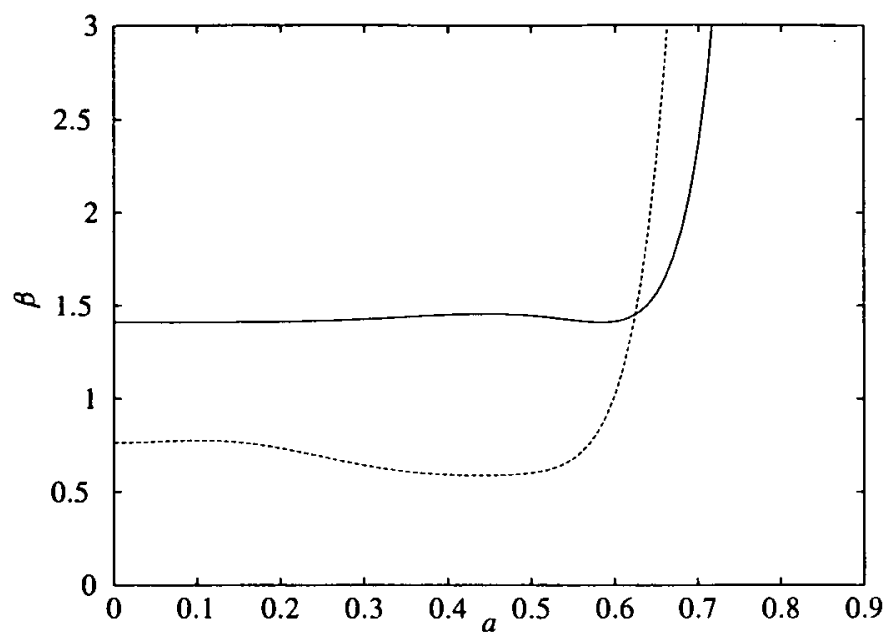

FIGURE 4. Power versus aperture opening: we show the curves for two short positions, $d=2.07$ (solid curve) and $d=2.62$ (dashed curve).

the empty, nearly closed, cavity resonant; hence even when it is loaded with a lossy slab it is more efficient for large $a$ that the cavity be shorted at $d=2.62$. In the no-iris limit $|\psi|=1$, and the difference in power required between the two choices of short position is due to slab placement. As the slab, of length two, is shorter than the distance, $\pi k_{r}^{-1}=2.54$, between the electric-field amplitude maxima in the cavity, the slab position, relative to the short, is quite important. For $d=2.62$ the slab straddles an electric-field amplitude maximum; while for $d=2.07$ the maximum is not near the slab's centre.

The optimal aperture opening for $d=2.62$, at $a=0.44$, results in a power requirement of $\beta=0.59$, much lower (some $23 \%$ ) than the no-iris case and vastly superior to the minimum of the $d=2.07$ curve. Hence it can be seen that the use of an iris can significantly improve the efficiency of the microwave heating process.

\section{Acknowledgement}

The authors would like to thank an anonymous referee for some useful suggestions.

\section{References}

[1] J. M. Hill and T. R. Marchant, "Modelling microwave heating", Appl. Math. Modelling 20 (1996) $3-15$. 
[2] V. M. Kenkre, L. Skala, M. W. Weiser and J. D. Katz "Theory of microwave effects on atomic diffusion in sintering: basic considerations of the phenomenon of thermal runaway", in Microwave processing of materials II (eds. W. B. Snyder, W. H. Sutton, M. F. Iskander and D. L. Johnson), (Mater. Res. Soc. Proc. 189, Pittsburgh, 1990) 179-183.

[3] G. A. Kriegsmann, "Cavity effects in microwave heating of ceramics", SIAM J. Appl. Math. 57 (1997) 382-400.

[4] L. D. Landau and E. M. Lifshitz, Statistical physics (Pergamon Press, London, 1958).

[5] L. Lewin, Theory of waveguides (John Wiley, New York, 1975).

[6] B. Liu and T. R. Marchant, "The microwave heating of two-dimensional slabs with small Arrhenius absorptivity", IMA J. Appl. Math. 62 (1999) 137-166. 